\title{
Algoritmo concorrente com decisão suave para equalização em sistemas OFDM no domínio da freqüência
}

\author{
Fabbryccio A. C. M. Cardoso, Estevan M. Lopes e Dalton S. Arantes
}

\begin{abstract}
Resumo - Este artigo propõe um algoritmo concorrente para equalização semi-cega no domínio da freqüência para sistemas OFDM. O objetivo é aumentar a vazão dos dados transmitidos sem prejuízo no desempenho do sistema, quando comparado com técnicas convencionais de estimação de canal baseadas em portadoras pilotos. A proposta é aplicar o conceito de equalização concorrente CMA+SDD a um banco de equalizadores no domínio da freqüência (pós FFT). O algoritmo proposto pode ser considerado semi-cego porque utiliza informação de subportadoras pilotos na inicialização, mantendo-se cego durante sua operação. Para suportar a equalização concorrente, o sistema deve prover pilotos apenas no primeiro símbolo de um superquadro, de modo a permitir que o algoritmo se inicialize quando o receptor for ligado ou quando a equalização for perdida. Nos demais símbolos do superquadro as portadoras pilotos são destinadas ao transporte de informação visando o aumento da vazão do sistema.
\end{abstract}

Palavras-Chave - Algoritmo concorrente, Equalização no domínio da freqüência, OFDM.

Abstract - This paper proposes a concurrent algorithm for semi-blind post-FFT equalization in OFDM systems. The objective is to allow an OFDM system design with increased data throughput, without performance loss when compared with pilot based conventional channel estimation techniques. This work explores the concept of CMA+SDD concurrent equalization to develop a frequency domain (post FFT) equalizer bank. The algorithm can be considered semi-blind because it uses channel information, obtained from pilot subcarriers, to initialize the equalizer bank, which remains blind during the equalization process. To support such concurrent equalization, the system should provide pilot subcarriers in the first symbol of an OFDM super-frame, allowing algorithm initialization when the receiver is turned on or when it loses equalization. In the remaining super-frame symbols the pilot subcarriers are used for data transmission in order to increase system throughput.

Keyword - Concurrent Equalization, Frequency domain equalization, OFDM.

Fabbryccio A. C. M. Cardoso e Dalton S. Arantes, Departamento de Comunicações, Faculdade de Engenharia Elétrica e de Computação, Universidade Estadual de Campinas (UNICAMP), Campinas, São Paulo, emails: [cardoso, dalton]@decom.fee.unicamp.br. Estevan M. Lopes, Instituto Nacional de Telecomunicações (Inatel), Santa Rita do Sapucaí, Minas Gerais, e-mail: estevan@inatel.br.

\section{INTRODUÇÃO}

Esquemas de equalização concorrente são uma solução eficiente e automática de se combinar o CMA - Constant Modulus Algorithm [1] com algoritmos baseados em decisão de símbolos de uma constelação de sinais, tais como o DD - Decision Direct [2] ou o SDD - Soft Decision Direct [3]. Desde a primeira publicação do Algoritmo Concorrente por De Castro et al. em 2001 [2], vários trabalhos se seguiram, fundamentados no conceito original de concorrência proposto em [2]. Em geral, esses trabalhos posteriores focaram nos aspectos mais expressivos do algoritmo, tais como baixo error floor, capacidade de recuperação de fase da portadora (a menos de uma ambigüidade de múltiplos de $\pi / 2$ ) e na alta velocidade de convergência. Dos trabalhos que se seguiram, vale destacar o de Chen [3], que em 2004 propôs um esquema concorrente $\mathrm{CMA}+\mathrm{SDD}$ com complexidade equivalente ao CMA+DD, porém com velocidade de convergência ainda mais rápida para os casos estudados.

Outras derivações do Concorrente podem ser citadas. Por exemplo, Shun-lan e Ming-zeng [4] propôs uma nova formulação com o equalizador MSCA (Modified Square Contour Algorithm) em substituição ao CMA, enquanto Rao [5] propôs o uso de um VSCMA (Vector Constant Modulus Algorithm).

Aplicações do equalizador concorrente em sistemas de comunicação já começam a surgir. Tipicamente, o algoritmo precisa ser adequado às especificidades do sistema ao qual será aplicado. Por exemplo, Hadef [6] utilizou uma variação do Concorrente para redução da ISI (Inter-Symbol Interference) e, conseqüentemente, da interferência de múltiplo acesso para sistemas DS-CDMA(Direct Sequence - Code Division Mutiple-Access) com detecção multiusuário. O concorrente também foi ligeiramente modificado por Rao et al [5] para se adequar a aplicações de transmissão de dados por propagação acústica em ambientes aquáticos variantes no tempo.

Seguindo a linha de aplicações do Concorrente em sistemas de comunicação, este artigo emprega o algoritmo CMA+SDD proposto por Chen [3] para equalização no domínio da freqüência (pós FFT) em sistemas OFDM. O objetivo é aumentar a vazão ou o desempenho de BER×SNR do sistema quando comparado com técnicas convencionais de estimação de canal, baseadas em portadoras pilotos e técnicas de interpolação.

Os sistemas convencionais de transmissão OFDM são concebidos para oferecer dois tipos de proteção contra as imperfeições na resposta impulsiva do canal de comunicação. A primeira proteção é realizada no domínio 
temporal, sendo denominada de prefixo cíclico, cuja finalidade é evitar que ocorra a interferência entre os símbolos OFDM. A segunda é uma proteção no domínio da freqüência empregando técnicas de estimação de canal e equalização.

O sistema de equalização é projetado para operar no domínio da freqüência e tem o objetivo de corrigir as degradações na amplitude e na fase de cada subportadora de dados recebida em uma comunicação OFDM. Estas degradações, conhecidas como interferência intra-símbolo, são responsáveis pelo aumento na taxa de erro de bits. Em sistemas que empregam o receptor OFDM convencional, utilizam-se subportadoras pilotos para viabilizar a estimação do canal, em conjunto com técnicas de interpolação para solucionar o problema de seletividade do canal.

Embora as subportadoras pilotos transmitidas sirvam para estimar a resposta em freqüência do canal de comunicação, a vazão do sistema é reduzida, uma vez que se deixa de transmitir dados nessas subportadoras. Observando esta limitação no sistema, propõe-se neste trabalho um algoritmo de equalização concorrente semi-cego capaz de recuperar as informações de amplitude e fase transmitidas, aumentando a vazão dos dados e melhorando a taxa de erro de bits quando comparado ao sistema de equalização convencional. O artigo está estruturado de forma a apresentar na Seção II o algoritmo proposto, a analisar na Seção III a vazão do sistema e os resultados de simulação e, finalmente, a apresentar na Seção IV as conclusões sobre o estudo.

\section{EQUAlizAdor CONCORRENTE SEMI CEGO}

\section{A. Equalizador Concorrente $C M A+S D D$}

O esquema de equalização concorrente mostrado na Figura 1 foi desenvolvido por De Castro et al. [2] e Chen [3] para um modelo de transmissão single-carrier com modulação QAM. O equalizador funciona com amostragem fracionária para poder operar em canais de fase não mínima [11]. As equações do algoritmo CMA+SDD desenvolvidas por Chen [3] são dadas por

$$
\begin{gathered}
y(n)=\mathbf{w}_{c}^{T} \mathbf{r}(n)+\mathbf{w}_{d}^{T} \mathbf{r}(n) \\
\mathbf{w}_{c}(2 n+1)=\mathbf{w}_{c}(2(n+1))=\mathbf{w}_{c}(2 n)+\mu \varepsilon(2 n) \mathbf{r}^{*}(2 n) \\
\varepsilon(2 n)=y(2 n)\left(\Delta_{2}-|y(2 n)|^{2}\right) \\
\Delta_{2}=\mathrm{E}\left\{|s(n)|^{4}\right\} / \mathrm{E}\left\{|s(n)|^{2}\right\} \\
\mathbf{w}_{d}(2 n+1)=\mathbf{w}_{d}(2(n+1))= \\
=\mathbf{w}_{d}(2 n)+\mu \frac{\partial \mathcal{J}_{L M A P}(\mathbf{w}, y(2 n))}{\partial \mathbf{w}_{d}} \\
\mathbf{w}(n)=\mathbf{w}_{c}(n)+\mathbf{w}_{d}(n)
\end{gathered}
$$

Como mostrado na Figura 1 e em (1), o equalizador concorrente é caracterizado pela adaptação independente de dois filtros FIR. Entretanto, pode-se mostrar que o mesmo algoritmo pode ser desenvolvido com um único filtro FIR adaptado concorrentemente pelos algoritmos CMA e SDD, como mostrado na Figura 2 e nas seguintes equações

$$
\begin{gathered}
y(n)=\mathbf{w}^{T}(n) \mathbf{r}(n) \\
\mathbf{w}(2 n+1)=\mathbf{w}(2(n+1))=\mathbf{w}(2 n)+\mu \varepsilon(2 n) \mathbf{r}^{*}(2 n) \\
\varepsilon(2 n)=y(2 n)\left(\Delta_{2}-|y(2 n)|^{2}\right) \\
\Delta_{2}=E\left\{|s(n)|^{4}\right\} / E\left\{|s(n)|^{2}\right\} \\
\mathbf{w}(2 n+1)=\mathbf{w}(2(n+1))= \\
=\mathbf{w}(2 n)+\mu \frac{\partial \mathcal{J}_{L M A P}(\mathbf{w}, y(2 n))}{\partial \mathbf{w}(2 n)}
\end{gathered}
$$

A versão do algoritmo concorrente mostrada em (2) foi adotada neste trabalho por economizar memória e também operações.

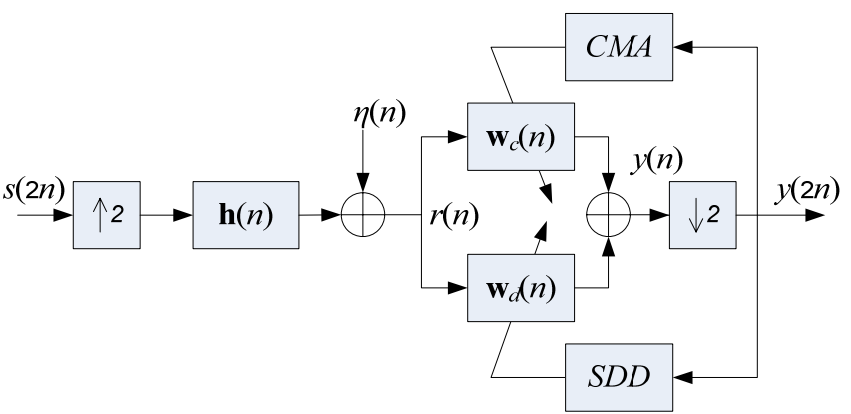

Figura 1. Modelo de comunicação em banda básica do equalizador concorrente.

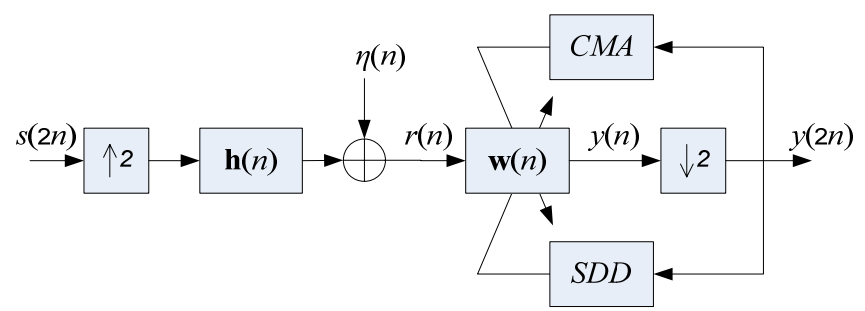

Figura 2. Modelo de comunicação em banda básica do equalizador concorrente modificado.

\section{B. Equalização Concorrente Aplicada a Sistemas OFDM}

A proposta inicial dos autores era projetar um algoritmo cego para um equalizador no domínio da freqüência que fosse capaz de recuperar as informações de amplitude e fase das portadoras transmitidas sem a utilização de portadoras pilotos como referência. Assim, o receptor OFDM, ilustrado na Figura 3, foi concebido para empregar um algoritmo de equalização concorrente com decisão suave, em um banco de equalizadores com um único coeficiente por subportadora.

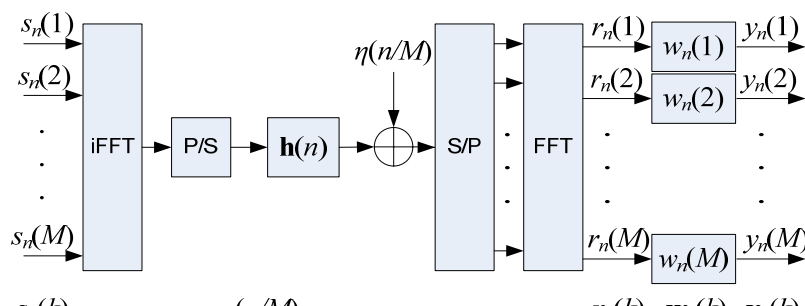

Figura 3. Modelo de comunicação em banda básica para sistema OFDM com banco de equalizadores $w_{n}(k)$.

O algoritmo projetado é baseado na técnica de equalização concorrente com decisão suave [3], onde os símbolos são equalizados de forma a atender ao critério MAP (Maximo A Posteriori)[12]. O modelo de equalização 
da Figura 3 pressupõe que o canal de comunicação pode ser representado, em sistemas OFDM, por $M$ canais de banda estreita, paralelos e ortogonais, onde não ocorre interferência mútua entre estes canais. Assim, cada subportadora é plana, ou seja, a faixa de cada subportadora é bem menor que a banda de coerência do canal. Neste artigo $\mathbf{w}_{n}(k)$ é o vetor de coeficientes $\left[w_{n}(k)\right]$ do banco de equalizadores, onde $k$ é o índice (subportadora) do vetor no instante $n$ e $M$ é a quantidade de subportadoras. $\mathrm{O}$ vetor com os dados da entrada e o vetor com os dados da saída do equalizador, são representados respectivamente por $\mathbf{r}_{n}(k) \mathrm{e}$ $\mathbf{y}_{n}(k)$. Observando-se a Figura 3, nota-se que a saída do banco de equalizadores pode ser expressa por

$$
y_{n}(k)=w_{n}(k) r_{n}(k) \text { para } k=1,2, \cdots, M
$$

A adaptação concorrente CMA+SDD de (2) foi aplicada ao banco de equalizadores $\boldsymbol{w}_{n}(k)$ com modificações. Observou-se que a equalização concorrente não consegue corrigir a fase da portadora quando há rotações de fase maiores do que $45^{\circ}$. Por este motivo, observou-se que a constelação recuperada, para as subportadoras com rotações de fase superiores a $45^{\circ}$, apresentavam, após a convergência dos equalizadores, rotações múltiplas de $90^{\circ}$ em tais cenários. Este resultado era esperado porque a recuperação da fase é possibilitada pela adaptação SDD, que se baseia em uma decisão suave da constelação do sinal recebido. Para exemplificar, considere um único símbolo de uma constelação 64 QAM, transmitido em todas as subportadoras com o valor $7+7$ i. A Figura 4 ilustra a composição dos sinais de todas as subportadoras de dados. Com a devida normalização em amplitude, observa-se que a fase do sinal pode variar bastante ao longo das subportadoras. Na Figura 4, para o perfil de canal Brasil A [13], constata-se que algumas subportadoras apresentam rotações maiores que $45^{\circ}$. Isto ocasiona uma elevação na taxa de erro de bits do sistema devido à convergência, em tais portadoras, para constelações com rotações múltiplas de $90^{\circ}$, sendo que o desejado seria $0^{\circ}$.

A solução proposta neste trabalho, para o problema de correção quando a rotação de fase é elevada, consiste em inicializar o banco de equalizadores com uma estimativa inicial do canal. O impacto dessa solução no sistema é a necessidade de se transmitir subportadoras pilotos, porém com uma freqüência muito menor que a necessitada por algoritmos tradicionais de equalização para sistemas OFDM. Como mostrado na Figura 5, a proposta é utilizar subportadoras pilotos apenas no primeiro símbolo de cada super-quadro. Além disso, no primeiro símbolo as pilotos estão espaçadas por subportadoras de dados de forma a aumentar ainda mais a vazão. Por este motivo, na inicialização dos equalizadores, correspondentes às portadoras de dados, é necessário interpolar as estimativas realizadas pelas pilotos.

Neste trabalho, uma vez inicializado o banco de equalizadores, o ajuste nos coeficientes é realizado de forma cega pelo concorrente CMA+SDD. A repetição das pilotos para cada primeiro símbolo do super-quadro é necessária para inicialização e sincronização do receptor quando o mesmo é ligado ou quando se perde a equalização. Esta proposta de equalização pode ser classificada como semicega devido ao emprego de informações do canal obtidas por pilotos apenas na fase de inicialização.
O esquema de equalização no instante inicial em que as subportadoras pilotos são utilizadas para estimar o canal de comunicação é ilustrado na Figura 6.

A inicialização do banco de equalizadores é descrita pela Equação 4, onde $P(k)$ é a piloto transmitida associada à $k$ ésima subportadora; $\widehat{P}(k)$ é a piloto recebida; e $w_{0}(k)$ é o valor inicial do coeficiente do equalizador associado à $k$ ésima subportadora OFDM. A interpolação é realizada entre as portadoras vizinhas no símbolo OFDM para inicialização de $w_{o}(k)$ quando $k$ corresponde a uma subportadora de dado.

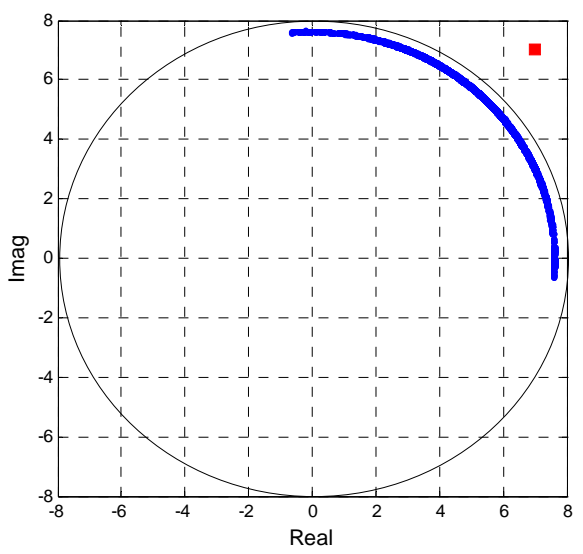

Figura 4. Constelação resultante da sobreposição das constelações das subportadoras do sinal OFDM recebido com normalização em amplitude do canal quando se transmite apenas o símbolo $7+7 i$.

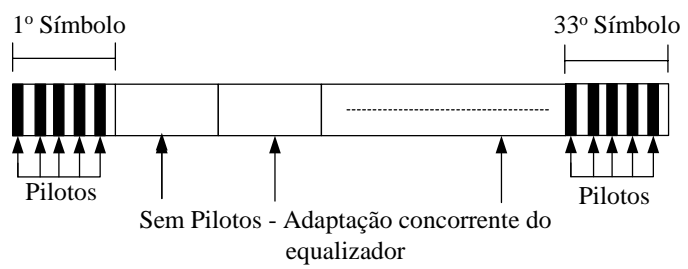

Figura 5. Utilização de subportadoras pilotos em super-quadros para inicialização do banco de equalizadores.

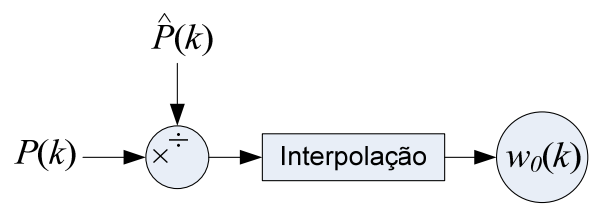

Figura 6. Diagrama de blocos da inicialização do banco de equalizadores.

$$
w_{0}(k)=\left\{\begin{array}{c}
\text { interp }\left\{\frac{P\left(m_{e}\right)}{\hat{P}\left(m_{e}\right)}, \frac{P\left(m_{d}\right)}{\widehat{P}\left(m_{d}\right)}\right\} \text { se } \\
k \in \text { subportadora de dados } \\
m_{e} \in \text { Piloto vizinha à esquerda } \\
m_{d} \in \text { Piloto vizinha à direita } \\
\frac{P(k)}{\hat{P}(k)} \text { se } \\
k \in \text { Piloto }
\end{array}\right.
$$

Outro aspecto importante que surge na aplicação da equalização concorrente em sistemas OFDM, além do aspecto da capacidade de correção de fase, é a perda da harmonia na operação concorrente do CMA e do SDD para subportadoras que foram bastante atenuadas pelo canal. Experimentalmente, observou-se que para atenuações de amplitude maiores que 0,1 (20dB), a atuação do SDD 
prejudica a atuação do CMA. Felizmente, a mesma solução de inicialização do equalizador adotada para o problema de ambigüidade de fase se aplica ao problema da perda de harmonia na concorrência entre o CMA e o SDD. De fato, a inicialização do equalizador a partir da estimação do canal provê uma condição inicial favorável para a atuação do concorrente.

A Tabela I resume o algoritmo concorrente final com as adequações sugeridas por este artigo para sua aplicação em sistemas OFDM. Vale destacar que nas simulações a adaptação do concorrente foi realizada a partir do gradiente estocático com passo normalizado pela potência média do sinal de entrada, semelhante ao NLMS. O fator de normalização de potência foi omitido no algoritmo da Tabela I por simplicidade. Os parâmetros do algoritmo empregados nas simulações foram $\mu_{c}=0,1, \mu_{d}=0,0001 \mathrm{e}$ $\rho=0,7$.

TABELA I

Algoritmo para equalização concorrente de sistemas OFDM.

Inicialização:

$$
w_{0}(k)=\operatorname{interp}(k, P(k), \hat{P}(k)),
$$

onde interp $(\cdot)$ é definida pela Equação 4.

Cálculo da saída no domínio da freqüência:

$$
y_{n}(k)=w_{n}(k) r_{n}(k)
$$

Atualização dos coeficientes do banco de equalizadores pelo concorrente $C M A+S D D$ :

CMA:

$$
\begin{gathered}
w_{n+1}(k)=w_{n}(k)+\mu_{c} \varepsilon_{n}(k) y_{n}(k) r_{n}^{*}(k) \\
\varepsilon_{n}(k)=\Delta_{2}-\left|y_{n}(k)\right|^{2} \\
\Delta_{2}=\frac{E\left\{\left|s_{n}(k)\right|^{4}\right\}}{E\left\{\left|s_{n}(k)\right|^{2}\right\}}
\end{gathered}
$$

$S D D[9][3]:$

$$
\begin{gathered}
w_{n+1}(k) \leftarrow w_{n+1}(k)+\mu_{d} \frac{\partial \mathcal{J}_{L M A P}\left(w, y_{n}(k)\right)}{\partial w} \\
(i, l)=\operatorname{encontra}\left(y_{n}(k)\right) \\
\frac{\partial \mathcal{J}_{L M A P}\left(w, y_{n}(k)\right)}{\partial w}= \\
\frac{\sum_{p=2 i-1}^{2 i} \sum_{q=2 l-1}^{2 l} \exp \left(-\frac{\left|y_{n}(k)-s_{p q}\right|^{2}}{2 \rho}\right)\left(s_{p q}-y_{n}(k)\right)}{\sum_{p=2 i-1}^{2 i} \sum_{q=2 l-1}^{2 l} \exp \left(-\frac{\left|y_{n}(k)-s_{p q}\right|^{2}}{2 \rho}\right)} r_{n}^{*}(k)
\end{gathered}
$$

\section{ANÁLISE DE RESULTADOS}

A metodologia empregada neste trabalho para avaliar a proposta de equalização Concorrente para sistemas OFDM consistiu em uma análise comparativa de desempenho de $\mathrm{BER} \times E_{b} / N_{0}$, tendo como referência os estimadores de Canal Conhecido e de Interpolação Linear. O estimador Canal Conhecido utiliza a informação conhecida do canal $C_{n}(k)$ nas subportadoras $k=1,2, \cdots, M$ para equalização, ou seja, para ajustar a amplitude e fase de cada subportadora $k$ por um fator $1 / C_{n}(k)$. Por ser ideal, a resposta de tal estimador deve representar uma referência de desempenho superior para o equalizador Concorrente, tanto de BER como de vazão. Por outro lado, o estimador Interpolação Linear utiliza pilotos fixas igualmente espaçadas por portadoras de dados para cada símbolo OFDM. A estimativa de canal de uma portadora piloto é calculada pela razão entre o sinal recebido e a piloto, ou seja,

$$
\hat{C}_{n}(k)=\frac{\hat{P}_{n}(k)}{P(k)}, \forall k \in \text { pilotos }
$$

Para as portadoras de dados, a estimativa de canal é obtida pela interpolação linear das estimativas obtidas das pilotos vizinhas.

Como a estimativa do estimador Interpolação Linear é utilizada para inicializar o banco de equalizadores e supondo que o processo de adaptação concorrente irá aprimorar a estimativa inicial, é razoável considerar que o desempenho do estimador Interpolação Linear seja um limitante inferior de desempenho para o Concorrente.

Neste trabalho, os resultados de simulação foram obtidos para um sistema OFDM com 2048 portadoras. Os símbolos OFDM, incluindo prefixo cíclico, possuem uma taxa de amostragem de $8,127 \mathrm{MHz}$, ou seja, um período de amostragem de $T_{s}=63 /\left(512 \times 10^{6}\right) \sim 123,05 n s$. Para formatação do espectro de freqüências, foram utilizadas, entre as 2048 portadoras, 158 portadoras nulas, restando 1890 para dados e pilotos. As portadoras de dados são moduladas com 64-QAM e as pilotos com BPSK.

Para o estimador Canal Conhecido o sistema é configurado sem portadoras pilotos sendo, portanto, a vazão do sistema $R_{\text {conh }}$, dada em bits/s, máxima e dada pelo inverso de $T_{s} \times 2048 \times(1+\mathrm{PC}) /(1890 \times 6)$. Para 0 estimador Interpolação Linear, o sistema é configurado com pilotos espaçadas entre si de 5 portadoras, resultando em 378 pilotos e 1512 portadoras de dados, em cada símbolo OFDM. Neste caso, a vazão do sistema $R_{\text {interp }}$ é dada pelo inverso de $T_{s} \times 2048 \times(1+P C) /(1512 \times 6)$. Para o equalizador concorrente, a configuração das pilotos é idêntica à do estimador Interpolação Linear apenas no primeiro símbolo do super-quadro. Nos demais símbolos do super-quadro todas as portadoras úteis, em um total de 1890, são utilizadas para dados. Neste caso, para um super-quadro contendo 32 símbolos, a vazão do concorrente $R_{\text {conc }}$ é dada pela média ponderada $R_{\text {conc }}=\left(1 \times R_{\text {interp }}+31 R_{\text {conh }}\right) /$ 32.

Neste trabalho, o algoritmo foi testado para valores de prefixo cíclico de 1/32 e 1/64. A Tabela II resume os valores de vazão sistêmica para os estimadores considerados neste trabalho. Vale destacar que não foi utilizado esquema algum de codificação de canal, visando enfatizar apenas o desempenho da equalização. Observe pela Tabela II que o concorrente possibilita uma economia de mais de $8 \mathrm{Mbits} / \mathrm{s}$ frente a sistemas que utilizam estimadores convencionais, como o estimador Interpolação Linear.

TABELA II

Vazão líquida de dados para os sistemas considerados na simulação.

\begin{tabular}{|l|l|l|l|}
\hline Receptor & $\begin{array}{l}\text { Canal } \\
\text { Conhecido }\end{array}$ & Concorrente & $\begin{array}{l}\text { Interpolação } \\
\text { Linear }\end{array}$ \\
\hline Vazão (Mbits/s) & & & \\
PC $=1 / 32$ & 43,64 & 43,36 & 34,91 \\
PC $=1 / 64$ & 44,31 & 44,03 & 35,45 \\
\hline
\end{tabular}

A escolha do prefixo cíclico de 1/32 e 1/64 foi motivada pelo perfil de canal utilizado nos testes. O objetivo é testar o 
equalizador concorrente no cenário em que o prefixo cíclico é suficiente para evitar interferência inter-simbólica (ISI) e em um cenário adverso, onde o prefixo cíclico não evita ISI. O perfil de canal utilizado nos testes é o ITU Brazil A [13], descrito na Tabela III. O ITU padroniza o perfil do canal em função do atraso e do ganho das componentes de multipercurso. A fase das componentes foi gerada aleatoriamente para simular o posicionamento da antena do receptor e foi mantida constante ao longo de todas as simulações. A digitalização do canal foi feita truncando para cima o perfil de atrasos em múltiplos do período de amostragem $T_{s}$. Os coeficientes não nulos do canal digitalizado são mostrados na Tabela III.

A duração do prefixo cíclico em $\mu s$ é dada, para os sistemas aqui considerados, por $2048 \times \mathrm{PC} \times T_{s}$. Isto corresponde, para $\mathrm{PC}=1 / 32$ e $1 / 64$, a durações de $7,875 \mu s$ e $3,9375 \mu s$, respectivamente. Portanto, como o maior atraso do canal é de $5,93 \mu s$, pode-se concluir que o prefixo cíclico de $1 / 32$ é suficiente para evitar ISI, enquanto que o prefixo de 1/64 não previne ISI, o que deverá degradar o desempenho do sistema. Neste trabalho, apresentamos resultados de desempenho de $B E R \times E_{b} / N_{0}$ para os cenários sem ISI $(P C=1 / 32)$ e com ISI $(P C=$ $1 / 64)$.

TABELA III

Perfil do canal ITU Brazil A

\begin{tabular}{|l|l|l|l|}
\hline $\begin{array}{l}\text { Coeficiente } \\
\text { ceil }\left(\text { Atraso } / T_{s}\right)+1\end{array}$ & Atraso $(\mu s)$ & Ganho (dB) & Fase (rad) \\
\hline 1 & 0,00 & 0 & 0,6 \\
\hline 3 & 0,15 & $-13,8$ & 3,4355 \\
\hline 20 & 2,22 & $-16,2$ & 1,9839 \\
\hline 26 & 3,05 & $-14,9$ & 4,2610 \\
\hline 49 & 5,86 & $-13,6$ & 5,6254 \\
\hline 50 & 5,93 & $-16,4$ & 0,0892 \\
\hline
\end{tabular}

Os resultados de simulação foram obtidos para cada ponto de $E_{b} / N_{0}$ pela estimativa da média $\xi$ da BER em $L=25$ realizações do experimento (simulação), como mostrado na Equação 6. A estimativa $S^{2}$ da variância e o cálculo do intervalo de confiança são mostrados pela Equação 7. Nos cálculos, supõe-se que $\overline{\mathrm{BER}}$ tem distribuição gaussiana e, portanto, que $(\overline{B E R}-\xi) / S \sqrt{L}$ tem distribuição t-student com $L-1$ graus de liberdade. No cálculo do intervalo de confiança IC de $90 \%$, o parâmetro $c$ é dado pela função inversa de distribuição acumulada $t$-student avaliada em 0,95 .

$$
\begin{gathered}
\overline{\mathrm{BER}}=\frac{1}{L} \sum_{l=1}^{L} \mathrm{BER}_{l} \\
S^{2}=\frac{1}{L-1} \sum_{l=1}^{L}\left(\mathrm{BER}_{l}-\overline{\mathrm{BER}}\right) \\
\operatorname{Pr}\left\{-c<\frac{\overline{\mathrm{BER}}-\xi}{S \sqrt{L}}<c\right\}=0,90 \\
\operatorname{Pr}\left\{\frac{\overline{\mathrm{BER}}-\xi}{S \sqrt{L}}<c\right\}=0,95 \\
\mathrm{IC}=[\overline{\mathrm{BER}}-c S \sqrt{L} ; \overline{\mathrm{BER}}+c S \sqrt{L}]
\end{gathered}
$$

As curvas de desempenho de $B E R \times E_{b} / N_{0}$ são então apresentadas com linha sólida para a estimativa da média $\overline{B E R}$ e com linha tracejada para os limites superior e inferior do intervalo de confiança IC.

A Figura 7 mostra os resultados de simulação para o cenário em que o prefixo cíclico é suficiente para evitar ISI, ou seja, para $P C=1 / 32$. Os resultados para o estimador Interpolação Linear saturam próximo a uma BER de $1 \times 10^{-3}$ devido à imprecisão da estimativa do canal nas portadoras de dados. Por outro lado, com o equalizador Concorrente, inicializado pela estimativa de canal do estimador Interpolação Linear, não ocorreu saturação na BER e o resultado acompanhou o comportamento do Canal Conhecido com uma perda da ordem de $5 \mathrm{~dB}$ nos pontos de $E_{b} / N_{0}$ avaliados.

Os resultados para o Cenário 2, onde o prefixo cíclico $\mathrm{PC}=1 / 64$ é insuficiente para evitar ISI, são apresentados na Figura 8. Neste cenário, a ortogonalidade entre as subportadoras é perdida e o modelo onde cada subportadora pode ser tratada como um canal independente não é mais válido. Ainda assim, os resultados obtidos pelo equalizador Concorrente foram melhores que os obtidos pelo estimador Interpolação Linear. Isto significa que o concorrente foi capaz de melhorar a estimativa de canal inicial realizada pela Interpolação Linear.

Para entender o comportamento do concorrente são apresentadas em azul, na Figura 9, as curvas de MSE de cada subportadora para $E_{b} / N_{0}=30 \mathrm{~dB}$. O primeiro destaque que se observa é o efeito de captura do concorrente relatado por De Castro et al. [2] e por Chen [3], quando a curva de MSE cai abruptamente. Também é notório que para algumas subportadoras o efeito de captura ocorre bem mais tarde do que para a maioria das subportadoras. A explicação para este fenômeno se deve à seletividade do canal que resulta em subportadoras com SNR muito baixa nas faixas de freqüência do canal que são afetadas por notches. De fato, o ruído térmico aditivo do receptor não é afetado pela seletividade do canal e a densidade espectral de potência do ruído permanece constante. Neste caso, se a resposta em freqüência do canal possui notches, as correspondentes subportadoras do sinal ficam inundadas por ruído. Conseqüentemente, a convergência do algoritmo é diretamente afetada pela SNR das subportadoras, explicando a maior lentidão na convergência de algumas delas. Mesmo com a lentidão na convergência de algumas sub-portadoras, a taxa de erro de bit média para este ponto de $E_{b} / N_{0}=$ $30 \mathrm{~dB}$ é da ordem de $1 \times 10^{-6}$.

\section{IV.CONCLUSÕES}

A proposta deste trabalho é utilizar adaptação concorrente CMA+SDD em um banco de equalizadores para correção cega de amplitude e fase das sub-portadoras de sistemas OFDM. A utilização do algoritmo concorrente foi motivada por ser um algoritmo cego de baixa complexidade que possui convergência bem mais rápida do que a do CMA. Outra vantagem do concorrente está na capacidade de recuperação da fase da portadora, que é inerente à adaptação do SDD.

Este trabalho contribuiu com a pesquisa de receptores OFDM, possibilitando o aumento na vazão do sistema através de uma redução drástica do uso de subportadoras piloto. Esta contribuição consistiu na aplicação e adaptação do algoritmo concorrente CMA+SDD para sistemas OFDM. Adicionalmente, também foi mostrado que a arquitetura do 
equalizador pode ser modificada para utilizar um único filtro FIR com adaptação concorrente, em vez de dois filtros concorrentes.

Na solução proposta, sugere-se que o banco de equalizadores seja inicializado a partir de uma estimativa preliminar do canal. O objetivo é reduzir as rotações de fase nas saídas do banco de equalizadores para valores menores do que $45^{\circ}$. Além disso, a inicialização também reduz a atenuação excessiva do canal nas subportadoras afetadas pela seletividade em freqüência. Esta medida foi tomada para evitar situações onde o SDD pode prejudicar a atuação do CMA. A contrapartida sistêmica para viabilizar a implementação desta inicialização é a inclusão de pilotos em um único símbolo de cada superquadro. Após a inicialização as subportadoras piloto não são mais utilizadas.

O desempenho do sistema foi avaliado em um ambiente de propagação modelado pelo perfil de canal ITU Brazil A. Os resultados mostraram que nas situações onde o prefixo cíclico é suficiente para evitar ISI, o desempenho do sistema acompanha o modelo de Canal Conhecido com uma perda de $\sim 5 \mathrm{~dB}$. No cenário com ISI, apesar do modelo multicanal ortogonal não ser mais válido, os resultados mostraram que a equalização concorrente melhora de forma significativa a estimativa inicial do canal.

\section{REFERENCES}

[1] C. Richard Johnson, Jr. et al., "Blind Equalization Using Constant Modulus Criterion: A review”, Proceedings of the IEEE, Vol. 86, No. 10, October 1998

[2] Fernando C. C. de Castro, Maria C. F. de Castro and Dalton S. Arantes, "Concurrent Blind Deconvolution for Channel Equalization", IEEE International Conference On Communications ICC2001, pp. 366-371, Helsinki, Finland, June 2001.

[3] S. Chen, "Low Complexity Concurrent Constant Modulus Algorithm and Soft Decision Directed Scheme for Blind Equalization”, IEE Proc. - Vis. Image Signal Processing, Vol 150, No. 5 October 2003.

[4] L. Shun-lan and D. Ming-zeng, "A New Blind Equalization Scheme for QAM Systems Based on Fractionally-Spaced Equalizer”, IEEE International Conference on Communications, Circuits and Systems, ICCCAS, Kokura, Japan, July, 2007.

[5] W. Rao, Y. Han and Y. Guo, "A New Family of Combination Blind Equalization with a New Constant Modulus Algorithm Based on Variable Slope Error Function", The $8^{\text {th }}$ International Conference on Signal Processing, ICSP2006 Proceedings, Beijing, 2006.

[6] M. Hadef, S. Weiss, "A Fast Converging Concurrent Affine Projection Algorithm for Blind Multiuser Equalisation”, The $2^{\text {nd }}$ IEE/EURASIP Conference on DSPenabledRadio, University of Southampton, Sept. 2005.

[7] Fernando C. C. de Castro, "Desconvolução autodidata concorrente para equalização de canais de comunicações", Tese de Doutorado, Universidade Estadual de Campinas - UNICAMP, Campinas, SP, Brasil, março 2001.

[8] S. Chen, E. S. Chang, "Blind FIR Equalization for High-Order QAM Signaling”. Signal Processing, $6^{\text {th }}$ International Conference, Pequim, Aug. 2002, Issue 26-30, Volume 1, pages 1299-1302.

[9] S. Chen, S. McLaughlin, P.M. Grant and B. Mulgrew, “ MultiStage Blind Clustering Equaliser", IEEE Transactions on Communications, Vol. 43, No. 2/3/4, February/March/Abril/ 1995.

[10] T. C. Hewavithana, and D. M. Brookes, "Blind Adaptive Channel Equalization for OFDM Using the Cyclic Prefix Data”, IEEE Communications Society, Globecom 2004.

[11] S. Chen, E. S. Chng, "Concurrent Constant Modulus Algorithm and Soft Decision Directed Scheme for Fractionally-Spaced Blind Equalization”, Proc. of IEEE Int. Conf. on Communications, Vol. 4, pp. 2342-2346, 2004.

[12] Simon Haykin, “Adaptive Filter Theory”, 3rd ed., Prentice Hall, Upper Saddle River, New Jersey, 1996.

[13] ITU Radiocommunication Study Groups: Document 6E/TEMP/131-E, Guidelines And Techniques For The Evaluation Of DTTB Systems, 19 March 2003.

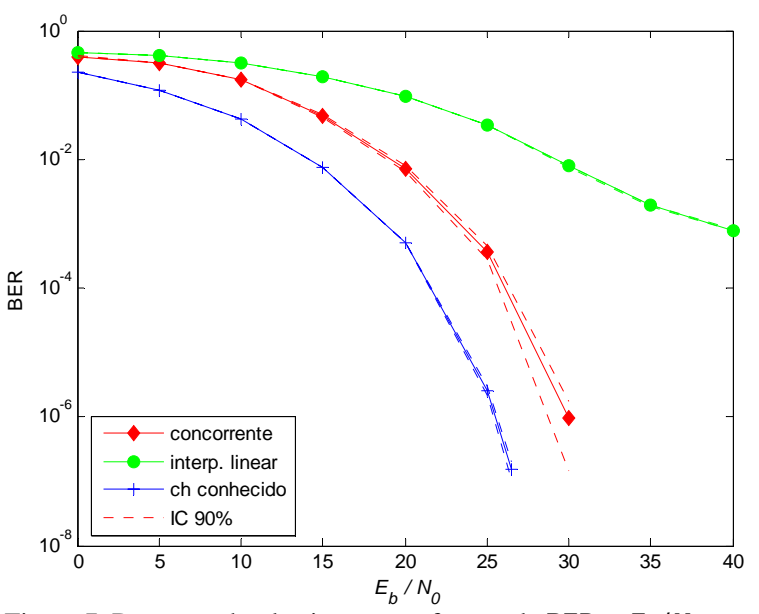

Figura 7. Desempenho do sistema em função de BER $\times E_{b} / N_{0}$ para o cenário de transmissão com $\mathrm{PC}=1 / 32$ suficiente para prevenir ISI. O modelo de canal utilizado é o ITU Brazil A.

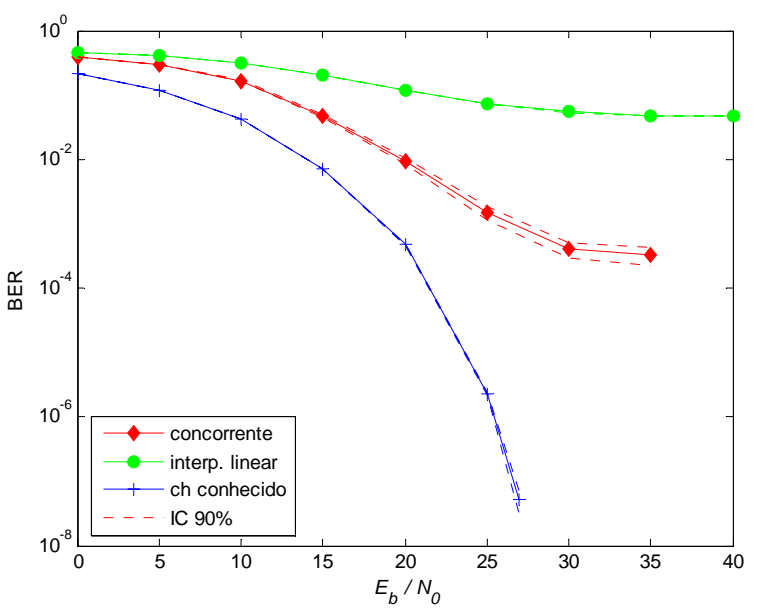

Figura 8. Desempenho do sistema em função de BER $\times E_{b} / N_{0}$ para o cenário de transmissão com $\mathrm{PC}=1 / 64$ insuficiente para prevenir ISI. O modelo de canal utilizado é o ITU Brazil A.

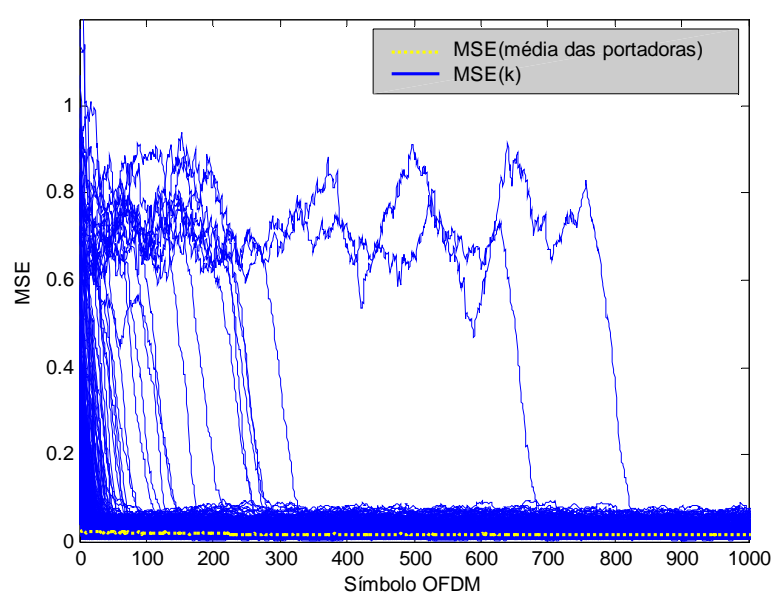

Figura 9. Curvas de erro quadrático médio $\operatorname{MSE}(k)$ por subportadora $k$, com $k=1,2, \cdots, 1890$. As curvas de $\operatorname{MSE}(k)$ são mostradas nas linhas em azul, na linha tracejada em amarelo é mostrada a média das curvas de $\operatorname{MSE}(k)$, ou seja, $\operatorname{MSE}=\sum_{k=1}^{1890} \operatorname{MSE}(k) / 1890$. 